\title{
THE DESCENDING BRANCH OF THE LATERAL FEMORAL CIRCUMFLEX ARTERY FOR CORONARY ARTERY BYPASS GRAFTING
}

Yuzuru Sakakibara, MD, Masakazu Abe, MD, Yuji Hiramatsu, MD, Osamu Shigeta, MD, Shigemi Ishikawa, MD, Tomoaki Jikuya, MD, and Toshio Mitsui, MD, Tsukuba, Japan

Tatsumi and associates ${ }^{1}$ attempted to use the lateral femoral circumflex artery (LFCxA) as a free graft for coronary bypass $(\mathrm{CABG})$ surgery. However, the LFCxA may play an important role as a collateral root in patients with femoropopliteal occlusive disease (arteriosclerosis obliterans). ${ }^{2}$ Both the applicability of the LFCxA as a bypass conduit and the suitability of sacrificing it at the risk of limb ischemia have to

From the Department of Surgery, Institute of Clinical Medicine, University of Tsukuba, Tsukuba, Japan.

Received for publication May 5, 1999; accepted for publication June 3, 1999.

Address for reprints: Y. Sakakibara, MD, Department of Surgery, Institute of Clinical Medicine, University of Tsukuba, Tsukuba Science City, Ibaraki, 305-8575, Japan.

J Thorac Cardiovasc Surg 1999;118:753-4

Copyright $@ 1999$ by Mosby, Inc.

$0022-5223 / 99 \$ 8.00+0 \quad \mathbf{1 2 / 5 4 / 1 0 0 6 1 4}$ be evaluated. We carried out an angiographic study to determine the suitability of the LFCxA as a free arterial graft for myocardial revascularization.

Patients and methods. Vascular records and panangiographic evaluations of the lower extremities from 1977 to 1989 were reviewed to identify all patients whose diagnosis of arteriosclerosis obliterans had been based on clinical characteristics and confirmed by angiography. Seventy-nine patients (120 limbs) were identified as having arteriosclerosis obliterans, meeting the conditions for precise measurement of LFCxAs in this study. Patients who had previous femoral artery surgery were excluded from this study. Seventy-one men and 8 women ranging from 40 to 83 years of age (mean 66.4 years) were examined. The maximal luminal diameter of the LFCxA was measured with a Digimatic caliper (Mitutoyo Corporation, Kawasaki, Kanagawa, Japan). The length of the LFCxA was measured between the origin of a descending branch of the LFCXA and the point of inner luminal diameter of $1.5 \mathrm{~mm}$ ( $\mathrm{L}>1.5 \mathrm{~mm}$ ) (Fig 1). Data were expressed as 


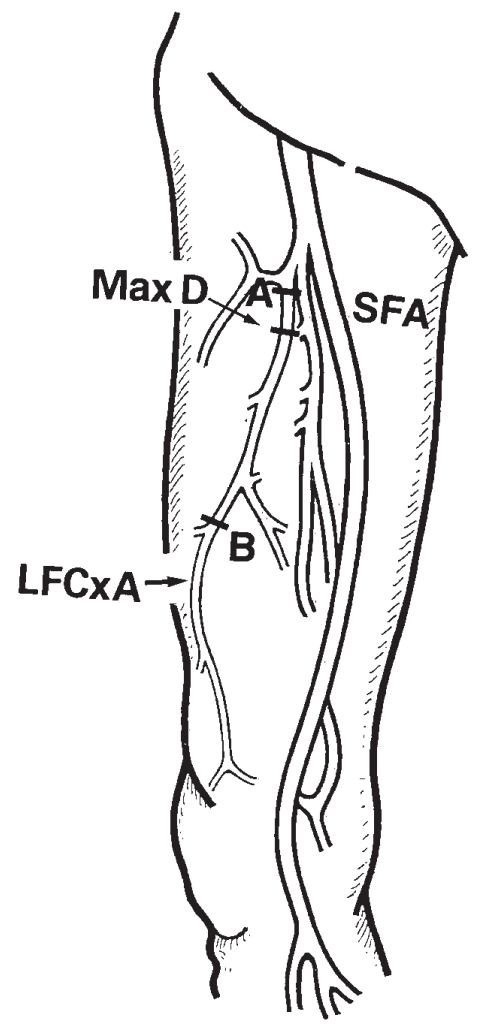

Fig 1. The descending branch of the lateral femoral circumflex artery ( $L F C x A)$. Max-D, Maximal inner diameter of the LFCxA (mm); SFA, superficial femoral artery. Straight line measurement of the length of the LFCxA pedicle from $(A)$ the origin of the descending branch of the LFCxA to $(B)$ the point of inner luminal diameter of $1.5 \mathrm{~mm}(\mathrm{~L}>1.5 \mathrm{~mm})$.

mean \pm standard deviation. The Student $t$ test was used for statistical analysis.

Results. On the basis of the arteriographic findings, these patients were divided into the following 2 groups: the collateral (+) group, in which a main collateral channel of the femoro-popliteal segment may play a significant role in distal circulation ( $\mathrm{n}=48$ limbs: $40 \%$ ), and the collateral (-) group, in which the LFCxA does not act as a major collateral vessel ( $n=72$ limbs: $60 \%$ ). The maximum inner diameter of the descending branch of the LFCXA was $2.06 \pm 0.55 \mathrm{~mm}$ in the collateral $(-)$ group and $2.95 \pm 0.66 \mathrm{~mm}$ in the collateral $(+)$ group $(P<.001) . \mathrm{L}>1.5 \mathrm{~mm}$ was $6.0 \pm 4.3 \mathrm{~cm}$ in the collateral $(-)$ group and $16.7 \pm 5.4 \mathrm{~cm}$ in the collateral $(+)$ group $(P<.001)$ (Table I).

Atherosclerotic changes of the LFCxA, which are indicated as wall irregularities, were recognized in 43 limbs $(35.8 \%$ of all those investigated). Useful LFCxAs for CABG free grafts (not a major collateral channel, without atherosclerotic change, more than $10 \mathrm{~cm}$ in pedicle length, and more than $1.5 \mathrm{~mm}$ in inner diameter) were recognized in only 12 limbs (10.0\%).

Discussion. Although many types of arterial graft materials are currently being used, surgeons are still searching for new
Table I. Measurement data for $L F C x A$

\begin{tabular}{lll}
\hline & Numbers or data & Percent \\
\hline Collateral (-) group & $72 \mathrm{limbs}$ & 60 \\
Max-D & $2.1 \pm 0.6 \mathrm{~mm}$ & \\
L $>1.5 \mathrm{~mm}$ & $6.0 \pm 4.3 \mathrm{~cm}$ & \\
Atherosclerotic change & $22 \mathrm{limbs}$ & 30.6 \\
Collateral (+) group & $48 \mathrm{limbs}$ & 40 \\
Max-D & $3.0 \pm 0.7 \mathrm{~mm}^{*}$ & \\
L $>1.5 \mathrm{~mm}$ & $16.7 \pm 5.4 \mathrm{~cm}^{*}$ & \\
Atherosclerotic change & $21 \mathrm{limbs}$ & $43.8(\mathrm{NS})$
\end{tabular}

$L F C x A$, Lateral femoral circumflex artery; $\operatorname{Max}-D$, maximum diameter of the descending branch of the LFCxA; $L>1.5 \mathrm{~mm}$, the length of the LFCxA pedicle with an inner diameter of more than $1.5 \mathrm{~mm}$; NS, not significant. Data are shown as the mean \pm the standard deviation. ${ }^{*} P<.001$.

grafts. ${ }^{3}$ Tatsumi and associates ${ }^{1}$ used the descending branch of the LFCXA as a free graft for CABG while making note of the large caliber and length of the LFCxA. Vaas, ${ }^{4}$ however, investigated the extent of atherosclerotic lesions of the LFCXA 1 $\mathrm{cm}$ beyond its origin using 100 cadavers of over 45 years of age. The percentage of narrowing at the investigated sites (LFCXA) was $23 \% \pm 22 \%$ in 56 men and $10 \% \pm 15 \%$ in 44 women. In his study, the percentages of atherosclerosis were not statistically different from those of the superficial femoral artery and those of the proximal portion of the deep femoral artery. Haimovici ${ }^{2}$ reported that the overall incidence of atherosclerotic changes in the profunda femoris was $9.5 \%$ in a nondiabetic group of patients and $30.5 \%$ in a diabetic group. In the present study, wall irregularities of the LFCxA were noted in $35.8 \%$ of all investigated limbs. Our study confirms the finding of a high incidence of atherosclerotic changes of the LFCxA in patients with arteriosclerosis obliterans.

Moreover, the profunda femoris and the descending branch of the LFCxA play an important role as a main collateral channel in patients with femoro-popliteal obstructive disease. In the present study, $40 \%$ of the total LFCxAs had become major collateral roots for peripheral circulation. Significantly larger calibers of LFCxAs were noted in the collateral (+) group than in the collateral (-) group. The LFCxAs with large diameters were potentially important collateral channels that could not be harvested. Our results suggest that lower extremity angiography should be performed in all patients in whom the use of the LFCxA as a graft is being considered.

\section{REFERENCES}

1. Tatsumi TO, Tanaka Y, Kondoh K, et al. Descending branch of lateral femoral circumflex artery as a free graft for myocardial revascularization. J Thorac Cardiovasc Surg 1996;112:546-7.

2. Haimovici H. Patterns of arteriosclerotic lesions of the lower extremity. Arch Surg 1967;95:918-33.

3. Foster ED, Kranc MAT. Alternative conduits for aortocoronary bypass grafting. Circulation 1989;79(Suppl):I34-9.

4. Vaas F. Atherosclerotic lesions at the bifurcation of the common femoral artery. Nether J Surg 1982;34:168-73. 